\title{
Review
}

\section{Minimal clinically important difference in idiopathic pulmonary fibrosis}

Idiopathic pulmonary fibrosis (IPF) is a chronic, progressive fibrosing lung disease with an estimated median survival of 2-5 years and a significant impact on quality of life (QoL). Current approved medications, pirfenidone and nintedanib, have shown a reduction in annual decline of forced vital capacity but no impact on QoL. The minimal clinically important difference (MCID) is a threshold value for a change in a parameter that is considered meaningful by the patient rather than solely relying on statistically significant change in the parameter. This review provides a brief overview of the MCID methodology along with detailed discussion of reported MCID values for commonly used physiological measures and patient-reported outcome measures in IPF. While there is no gold standard methodology for determining MCID, there are certain limitations in the MCID literature in IPF, mainly the choice of death, hospitalisation and pulmonary function tests as sole anchors, and pervasive use of distribution-based methods which do not take into account the patient's input. There is a critical need to identify accurate thresholds of outcome measures that reflect patient's QoL over time in order to more precisely design and evaluate future clinical trials and to develop algorithms for patient-oriented management of IPF in outpatient clinics.

\section{Educational aims}

- To understand the concept of MCID and the methods used to determine these values.

- To understand the indications and limitations of MCID values in IPF.

Idiopathic pulmonary fibrosis (IPF) is a chronic, progressive fibrosing lung disease with an estimated median survival of 2-5 years and a significant impact on quality of life (QoL) [1]. The two US Food and Drug Administration (FDA) approved treatments available for IPF, pirfenidone and nintedanib, have shown a reduction in the annual rate of decline of forced vital capacity (FVC) in clinical trials [2-6]. However, neither medication has shown a difference in QoL as measured by two validated

@ERSpublications

Minimal clinically important difference (MCID) values for common physiological and patientreported outcome measures have been estimated in IPF, but have limitations. MCID research can help advance patient-oriented management of IPF. https://bit.ly/314Q5iH

Cite as: Kang M, Marts L, Kempker JA, et al. Minimal clinically important difference in idiopathic pulmonary fibrosis. Breathe 2021; 17 : 200345. 
instruments, the University of California, San Diego Shortness of Breath Questionnaire (UCSD SOBQ) and the St. George's Respiratory Questionnaire (SGRQ), which were secondary outcomes in the trials for pirfenidone and nintedanib, respectively [3, 5-7]. This incongruity between objective measures of lung function and subjective, patientreported QoL metrics highlights a limitation in the translation of IPF research to clinical care. This dichotomy in trial results may be due to the lack of validity and responsiveness of QoL measures or perhaps because the difference in decline of FVC in the trials does not reflect patient-centred outcomes. Therefore, there is a critical need for robust estimates of the minimal clinically important difference (MCID) of key objective measures that correspond to improvements in patient QoL to help guide clinical care decisions and evaluate outcomes of clinical trials in the field of IPF.

Annual rate of decline in FVC has been used as a surrogate marker in major clinical trials [2-6]. While mortality and hospitalisation are inherently meaningful end-points for both clinicians and patients, they may not be feasible primary endpoints for IPF trials. When studying mild and moderate IPF, disease stages hypothesised to be both clinically detectable and susceptible to early therapy, there may be few deaths or hospitalisations within a feasible trial follow-up period. Therefore, to achieve statistical power, clinical trials with a primary end-point of death and/or hospitalisation would require large sample sizes that may be difficult to achieve in a rare disease [8]. This makes physiological measures of lung function more feasible alternative trial end-points, especially given their low cost and face validity as relevant markers for disease progression. However, in a disease that is nearly universally progressive and without corresponding improvements in QoL, it is uncertain whether statistically significant differences in these lung function measures are clinically meaningful to patients, especially given the high costs of medications and prevalence of side-effects. Rather than solely relying on statistically significant changes in a measure, MCID methodologies attempt to estimate a threshold value for a change in a measure that is considered important to patients. This review will provide a brief overview of the MCID concept and methodology along with detailed discussion of the MCID values of several physiological measures of lung function and patient-reported outcome measures (PROMs) in IPF that have been reported in literature.

\section{MCID methodology overview}

The MCID concept was introduced in 1989 by JAESCHKE et al. [9] and has traditionally been estimated by a triangulation of three approaches: 1) anchor-based methods, 2) distribution-based methods, and 3) expert opinion [10, 11]. Anchorbased methods determine an MCID by quantifying the minimal numerical change in a measure that is associated with a subjective, patientreported "anchor" estimation of improvement or worsening. While several anchor-based methods exist, estimates based on receiver operating curves are reported to be more accurate compared with mean change methods (table 1) [12].

Distribution-based methods, by contrast, rely on the statistical distribution of a measure to estimate the MCID. Given that distribution-based methods lack patient input in determining MCID estimates, they mainly measure the minimal detectable change (MDC), which is the minimum change that can be detected beyond measurement error [13]. MCID values estimated using anchor-based methods may fall on either side of the MDC. When MCID is lower than MDC, MCID is indistinguishable from measurement error yet still important [13].

Table 1 Summary of commonly used anchor-based methods

\begin{tabular}{|c|c|c|}
\hline Method & Explanation & Comment \\
\hline Jaeschke & Mean $\left(C_{\text {follow-up }}-C_{\text {baseline }}\right)$ & Regression to the mean \\
\hline $\begin{array}{l}\text { Mean change, } \\
\text { Redelmeier }\end{array}$ & $\begin{array}{l}\text { Mean }\left(C_{\text {follow-up }}-C_{\text {baseline }}\right)-\text { Mean } \\
\left(U_{\text {follow-up }}-U_{\text {baseline }}\right)\end{array}$ & Regression to the mean \\
\hline $\begin{array}{l}\text { Receiver operating } \\
\text { curve }\end{array}$ & $\begin{array}{l}\text { Cut-off point where sensitivity+specificity } \\
\text { is maximised between changed and } \\
\text { unchanged }\end{array}$ & $\begin{array}{l}\text { Non-parametric } \\
\text { Random sample variation } \\
\text { Change analysed separately for } \\
\text { worsening and improvement }\end{array}$ \\
\hline $\begin{array}{l}\text { Regression } \\
\text { modelling }\end{array}$ & $\begin{array}{l}\text { Transition variable } e^{\#} \text { as independent or } \\
\text { dependent variable }\end{array}$ & $\begin{array}{l}\text { Control for confounding and } \\
\text { effect modification }\end{array}$ \\
\hline
\end{tabular}

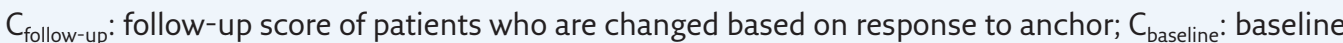
score of patients who are changed based on response to anchor; $\cup_{\text {follow-up }}$ : follow-up score of patients who are unchanged based on response to anchor; $U_{\text {baseline }}$ : baseline score of patients who are unchanged based on response to anchor. \#: transition variable (usually coded as 1 if changed based on response to anchor or 0 if unchanged based on anchor). 
MCID and not MDC is recommended to assess changes important to the patients [13].

Expert opinion is a subjective determination that incorporates clinical experience and may be conducted through a formal collective decisionmaking process such as the Delphi method or an informal value-based judgement. In the MCID studies of IPF patients reviewed in subsequent sections there are no explicit expert opinion-based estimates. However, expert judgements were used to determine the cut-off points of anchors in some studies and in deriving the final point-estimate from anchor-based and distribution-based methods.

Given that there is no current gold standard method to estimate MCID, there are several principles that need to be considered when evaluating proposed MCID values for a given condition. MCID values of the same measure may differ amongst different patient populations. For example, MCID values for SGRQ may vary for patients with COPD versus IPF. Even within the same disease, MCID values may vary by disease severity and follow-up time period, which can have important implications for clinical trial design. MCID values for improvement versus deterioration may also vary depending on the disease process and its natural history and should ideally be calculated and reported separately. Anchors such as death or hospitalisation may indicate maximal instead of minimal clinically important change. There is also concern about using pulmonary function tests as sole anchors for estimating MCID since they do not directly assess patient input about change in health status. Further research and standardisation of MCID methodology would help address these concerns and help advance the field of patient-oriented research.

\section{MCID values in IPF}

Ten studies have determined MCID values for various physiological measures and PROMs in IPF patients (table 2). In the following subsections we will address MCID estimates for each measure separately, summarising the evidence and assessing its strengths and limitations.

\section{Physiologic measures}

Forced vital capacity

To date, only one study from Du BoIs et al. [14] has estimated MCID values for FVC in IPF with data from 1156 patients enrolled in two interferon- $\gamma 1 \mathrm{~b}$ trials. The first trial enrolled 330 patients with FVC values of $50-90 \%$ predicted and $D_{\mathrm{Lco}}$ value $\geq 25 \%$ predicted [15]. The second trial included 826 patients with FVC between 55 and $90 \%$ predicted and $D_{\text {Lco }}$ between 35 and $90 \%$ predicted [16]. With these data, Du Bols et al. [14] reported an absolute change in FVC of $2-6 \%$ predicted over 48 weeks as the MCID value for both improvement and worsening in IPF.
This study incorporated a large sample size and the final MCID was determined using distribution and anchor-based methods. The authors chose several anchors: death, hospitalisation, composite of death and hospitalisation, and the health transition question in SF-36. The health transition question (SF-2) asks patients to rate their general health compared to 1 year ago on a 5-point Likert scale [17]. As stated previously, death and hospitalisation may be less than ideal anchors to assess minimal change, and distribution methods do not account for importance based on patient's input. Therefore, the final estimate of 2-6\% calculated by Du BoIs et al. [14] may not reflect the true MCID of FVC in IPF patients.

\section{6-min walking distance}

Three studies have reported MCID values for both worsening and improvement of 6MWD in IPF patients, each with different populations and anchors [18-20]. Du BoIs et al. [18] used the larger interferon- $\gamma 1 \mathrm{~b}$ trial cohort $(n=826$, FVC of $55-90 \%$ predicted and $D_{\text {LCO }} 35-90 \%$ predicted), and used hospitalisation, death and composite of death and hospitalisation as the only anchors. They calculated an MCID value of 24-45 m over 48 weeks using distribution and anchor-based methods. NATHAN et al. [19] used data from 338 IPF patients in the CAPACITY trials of pirfenidone with FVC $\geq 50 \%$ predicted and $D_{\text {LCO }} \geq 35 \%$ predicted. Using distribution-based methods and anchors of hospitalisation, death and a composite of both, they estimated an MCID in the range of 21.737.0 m over 48 weeks. SwIGRIs et al. [20] used the BUILD-1 study cohort, which investigated the use of Bosentan in 123 IPF patients with FVC of $50-90 \%$ predicted and $D_{\mathrm{LCO}} \geq 30 \%$ predicted. They averaged the estimates obtained from both distribution and anchor-based methods for a final MCID estimate of $28 \mathrm{~m}$ over 52 weeks using FVC and SGRQ as anchors. This may not yield an accurate estimate of MCID since distribution-based methods and anchor-based methods were weighted equally. It is important to note that all three studies with different time periods and anchors estimated similar MCID values for 6MWD ranging from 21.7 to $45 \mathrm{~m}$.

\section{Incremental shuttle walk}

While 6MWD is more commonly used, ISW is another measure of a patient's physical function [21]. NolAn et al. [21] determined MCID in 77 patients after an 8-week pulmonary rehabilitation programme with no specific inclusion criteria for FVC or $D_{\mathrm{LCO}}$. They reported MCID as a range of 31-46 m over 8 weeks using distribution and anchor-based methods. Since these patients were undergoing a pulmonary rehabilitation programme, most of the respondents had an improvement in their anchor, GRCQ, which rated how they felt overall on a 5-point Likert scale. Therefore, the reported MCID value is 
Table 2 Summary of MCID values of physiological and patient-reported outcome measures in patients with IPF

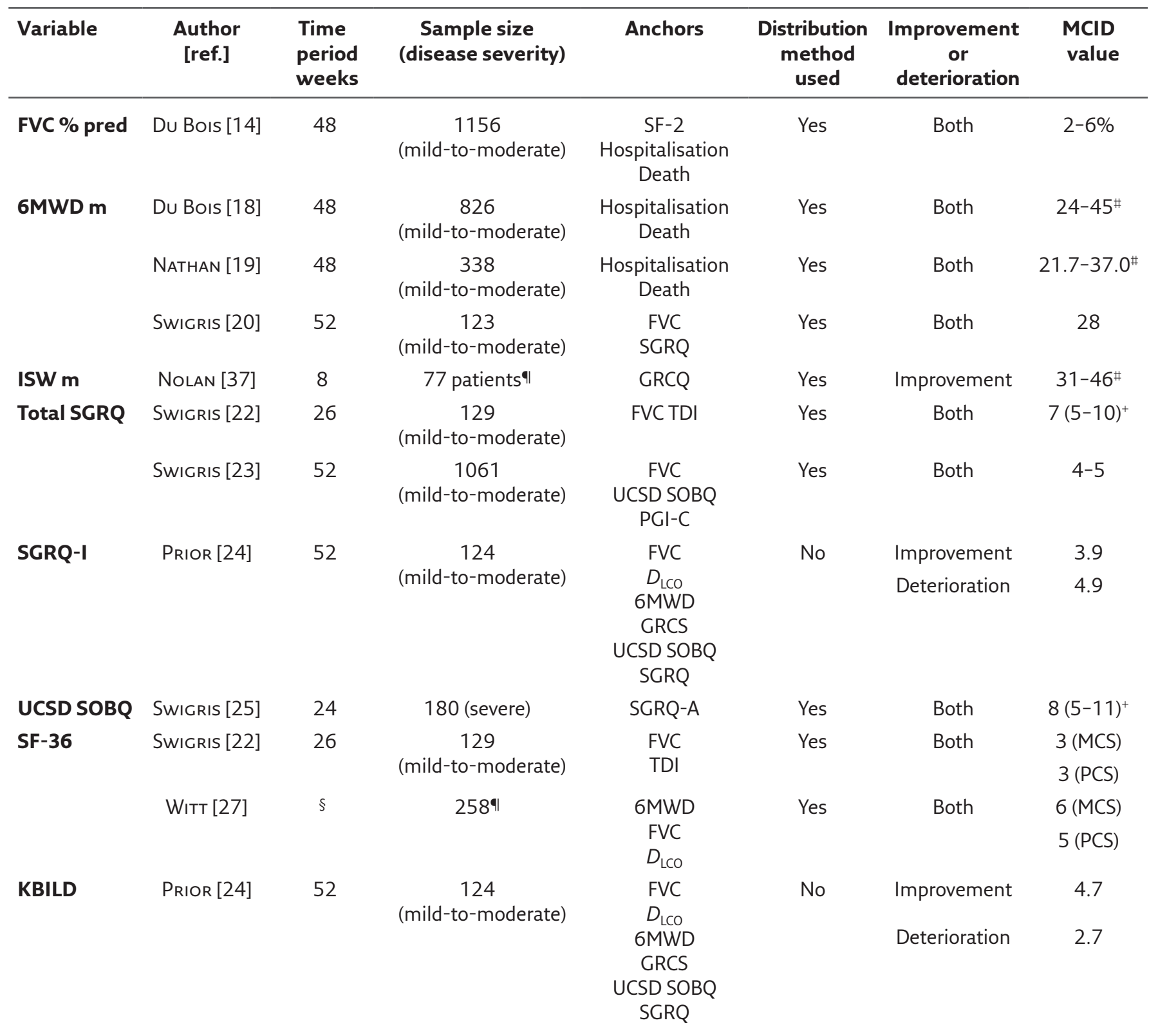

SGRQ-I: SGRQ for patients with IPF; SF-36: Short-Form Health Survey 36; SF-2: health transition question in SF-36 questionnaire; GRCQ: Global Rating of Change Questionnaire; TDI: Transition Dyspnea Index; PGI-C: Patient's Global Impression of Change; $D_{\text {Lco: }}$ diffusing capacity of the lung for carbon monoxide; GRCS: Global Rating of Change Scales; SGRQ-A: SGRQ activity domain; MCS: mental component score; PCS: physical component score. \#: range; १: no set inclusion criteria based on disease severity; ${ }^{+}$: point estimate (range); §. no a priori follow-up time.

most applicable for improvement and may not reflect worsening in patients with IPF.

\section{Patient-reported outcome measures}

St. George's Respiratory Questionnaire

As stated previously, SGRQ has been used as an end-point to assess patient QoL in IPF clinical trials but it was originally developed for patients with obstructive lung diseases [3]. SWIGRIs et al. [22] estimated the MCID of total SGRQ score using the BUILD- 1 trial ( $\mathrm{n}=129$, FVC of $50-90 \%$ and $D_{\mathrm{LCO}}$ $\geq 30 \%$ ) and reported an MCID of 7 points (range 5-10) over 26 weeks by averaging estimates from both distribution and anchor-based methods with FVC and TDI as anchors. A separate study by the same first author used a pooled cohort from the INPULSIS trials of nintedanib in patients with FVC 
$\geq 50 \%$ predicted and $D_{\text {LCO }} 30-79 \%$ predicted [23]. The authors used FVC, UCSB SOBQ, and PGI-C as anchors and determined a range of 4-5 points as the lower threshold of MCID over a 52-week period [23].

The MCID of an IPF specific version of this questionnaire, SGRQ-I, was evaluated as a primary objective by PRIOR et al. [24] in 124 IPF patients (no specific inclusion criteria, but baseline characteristics showed mild-to-moderate impairment in $\mathrm{FVC}$ and $D_{\mathrm{LCO}}$ ). An MCID of 3.9 points for improvement and 4.9 points for worsening was determined using anchor-based methods with six anchors: FVC, $D_{\mathrm{LCO}}, 6 \mathrm{MWD}$, SGRQ, UCSD SOBQ, and GRCS [24]. The separation of MCID values according to improvement and worsening is a better method to evaluate changes in a progressive disease where most patients would be expected to fall in the category of either unchanged SGRQ-I scores or worsening scores over the course of a specific time period even while on treatment with antifibrotic medications.

University of California, San Diego Shortness of Breath Questionnaire

UCSD SOBQ was used to assess patient QoL as a secondary end-point in the CAPACITY and ASCEND trials of pirfenidone [5, 6]. SwIGRIs et al. [25] determined an MCID value using a cohort of patients from the STEP-IPF trial studying sildenafil in patients with IPF with $D_{\mathrm{LCO}}<35 \%$ predicted. The study determined an MCID value of 8 points over 24 weeks by averaging values ranging from 5-11 obtained from anchor and distribution-based methods. The single anchor used in this study was the activity domain of SGRQ (SGRQ-A), which provided an MCID estimate of 5-9 points [25].

\section{Short-form health survey 36}

SF-36 is a general health survey validated in IPF patients [26]. Scores are often reported as two separate components: physical component score (PCS) and a mental component score (MCS). WITT et al. [27] reported an MCID of 5 for PCS and 6 for MCS in 258 patients enrolled in the European IPF registry with varying disease severity by using anchor and distribution-based methods. Their sole use of FVC, $D_{\mathrm{LCO}}$ and 6MWD as anchors, however, limited patient input into MCID determination. Additionally, the study did not have an a priori follow-up time and measures were assessed at different time periods with an average follow-up time of 1.3 years [27]. The study of MCID in SGRQ by SwIGRIs et al. [22] evaluated MCID for SF-36 in patients in the BUILD-1 trial $(n=129$, FVC of $50-90 \%$ predicted and $D_{\text {LCO }} \geq 30 \%$ predicted) and used FVC and TDI as anchors along with distribution-based methods. They reported PCS and MCS values of 3 over a 6 -month time period as the MCID.

\section{King's Brief Interstitial Lung Disease}

The KBILD questionnaire was designed specifically for patients with interstitial lung disease (ILD), including IPF, unlike the other PROMs previously discussed [28]. KBILD was the secondary end-point in a recent trial evaluating nintedanib in patients with progressive ILD [29]. The KBILD calculation was mathematically standardised using a new logit-transformed scale around 2010 [30]. The only study evaluating MCID values of KBILD in IPF patients, by PrIOR et al. [24], was conducted using the logit-transformed scores. The authors used anchor-based methods, notably using six anchors: FVC, $D_{\mathrm{LCO}}, 6 \mathrm{MWD}, \mathrm{SGRQ}$, UCSD SOBQ, and GRCS. This study reported different values for improvement (4.7 points) and worsening (2.7 points) over a 52-week time period in 124 patients with no specific inclusion criteria, but baseline characteristics showed mild-to-moderate impairment in FVC and $D_{\mathrm{LCO}}$ [24].

\section{Conclusion and future directions}

There are reported MCID values for commonly used physiological measures, such as FVC and 6MWD, and PROMs, such as SGRQ, UCSD SOBQ, SF-36 and KBILD, in IPF. While there is no gold standard methodology for determining MCID, there are certain limitations in the MCID literature in IPF, mainly the choice of death, hospitalisation and pulmonary function tests as sole anchors, pervasive use of distribution-based methods which do not take into account the patient's input, and averaging estimates from different methods to arrive at a single point MCID estimate. Therefore, the MCID values reviewed in this article may be used with caution when designing or evaluating clinical trials.

PROMs can measure a variety of constructs such as dyspnoea, physical functioning, mental health, etc. Patients' QoL may be influenced by factors unrelated to the disease itself such as other health comorbidities, treatment adverse effects, socioeconomic stressors, and depression. Well designed and validated PROMs can assess various QoL domains more objectively and can guide management decisions along with physiological measures. While there is some evidence of convergent validity and data on psychometric properties of non-disease specific questionnaires such as SGRQ, UCSD SOBQ and SF-36 in IPF, further confirmatory studies on content and longitudinal validity are needed for their continued use as outcome measures in clinical trials and for unbiased MCID estimation [22, 23, 25, 31-33]. A validated composite outcome measure to monitor disease progression that incorporates lung function, physical activity measures and PROMs, like the BODE index in COPD, could guide therapy in clinical practice and may also be used as an end-point in 
research trials [34]. To our knowledge, there has been no such measure developed in IPF, although composites of only clinical and physiological measures, such as the GAP (gender $(G)$, age $(A)$ and two lung physiology $(\mathrm{P})$ variables: FVC and $\left.D_{\mathrm{LCO}}\right)$ index and composite physiologic index (CPI), have been developed to predict mortality [35, 36].

This review highlights that there is much work to be done in the MCID domain to set a consistent, patient-centred, and robust research agenda for future clinical trials and clinical care in IPF. In our opinion, such research directions include: 1) MCID estimation in IPF patients with varying severity and over varying time periods with novel anchor-based methods; 2) determination of psychometric properties and MCID values of commonly assessed measures such as total lung capacity, Borg dyspnoea scale, and Euroqol questionnaires in the IPF population; 3) further study of outcome measures and MCID values that can be applied to individual patients in clinical settings; and 4) development of well-validated and reliable physiological outcomes, IPF-specific PROMs, and a composite of physiological and patient-reported outcomes, which may better capture the patient experience and help provide new clinical end-points for research studies and clinical care.

\section{Key points}

- There is no gold standard for MCID determination.

- Distribution-based methods, which do not incorporate the patient perspective, are not ideal for MCID calculation.

- Anchor-based methods using death or hospitalisation as anchors may estimate maximal and not necessarily minimal clinically important differences.

- Further research is needed to determine MCID values in IPF for different disease severity groups and follow-up time periods.

\section{Self-evaluation questions}

1. Which of the following is an anchor-based method used for estimating MCID values?
a) Regression modelling
b) Receiver operating curve
c) Mean change methods
d) All of the above

2. Which of the following is a limitation of using distribution-based methods in estimating MCID?

a) Distribution-based methods require expert consensus

b) Distribution-based methods incorporate patient input

c) Distribution-based methods estimate minimal detectable change but not minimal clinically important change

d) None of the above

3. What is the proposed MCID value of FVC over 48 weeks in IPF?
a) $7-10 \%$
b) $1-2 \%$
c) $2-6 \%$
d) $6-10 \%$

4. Which of the following is true regarding MCID?
a) MCID values may differ according to specific disease state
b) MCID values are always the same for worsening and improvement for any particular disease
c) MCID values estimated using consensus approach are superior to anchor-based methods
d) MCID values determined by averaging distribution- and anchor-based estimates are superior to anchor-based only methods. 


\section{Affiliations}

Mohleen Kang, Lucian Marts, Jordan A. Kempker, Srihari Veeraraghavan

Division of Pulmonary, Allergy, Critical Care and Sleep Medicine, Emory University School of Medicine, Atlanta, GA, USA.

\section{Conflict of interest}

M. Kang reports grants from National Heart, Lung, and Blood Institute (Division T32 grant: 5T32HL116271-07; PI Dr. David Guidot), during the conduct of the study. L. Marts reports grants from aTyr Pharma, outside the submitted work. J.A. Kempker reports grants from Agency for Healthcare Quality and Research (K08HS025240) and personal fees from Grifols, Inc, outside the submitted work. S. Veeraraghavan reports personal fees from Boehringer Ingelheim (Advisory board), and research support grants from Fibrogen, Bellerophon, Biogen, Nitto Denko, Pliant, Galapagos and Galecto, outside the submitted work.

\section{References}

1. Raghu G, Chen SY, Yeh WS, et al. Idiopathic pulmonary fibrosis in US Medicare beneficiaries aged 65 years and older: incidence, prevalence, and survival, 2001-11. Lancet Respir Med 2014; 2: 566-572.

2. Raghu G, Rochwerg B, Zhang Y, et al. An official ATS/ERS/ JRS/ALAT clinical practice guideline: treatment of idiopathic pulmonary fibrosis. An Update of the 2011 Clinical Practice Guideline. Am J Respir Crit Care Med 2015; 192: e3-e19.

3. Richeldi L, du Bois RM, Raghu G, et al. Efficacy and safety of nintedanib in idiopathic pulmonary fibrosis. N Engl J Med 2014; 370: 2071-2082.

4. Richeldi L, Costabel U, Selman M, et al. Efficacy of a tyrosine kinase inhibitor in idiopathic pulmonary fibrosis. N Engl/ Med 2011; 365: 1079-1087.

5. Noble PW, Albera C, Bradford WZ, et al. Pirfenidone in patients with idiopathic pulmonary fibrosis (CAPACITY): two randomised trials. Lancet 2011; 377: 1760-1769.

6. King TE, Jr, Bradford WZ, Castro-Bernardini S, et al. A phase 3 trial of pirfenidone in patients with idiopathic pulmonary fibrosis. N Engl J Med 2014; 370: 2083-2092.

7. Richeldi L, Cottin V, du Bois RM, et al. Nintedanib in patients with idiopathic pulmonary fibrosis: Combined evidence from the TOMORROW and INPULSIS((R)) trials. Respir Med 2016; 113: 74-79.

8. Collard HR, Brown KK, Martinez FJ, et al. Study design implications of death and hospitalisation as end points in idiopathic pulmonary fibrosis. Chest 2014; 146: 1256-1262.

9. Jaeschke R, Singer J, Guyatt GH. Measurement of health status. Ascertaining the minimal clinically important difference. Control Clin Trials 1989; 10: 407-415.

10. Jones PW, Beeh KM, Chapman KR, et al. Minimal clinically important differences in pharmacological trials. Am J Respir Crit Care Med 2014; 189: 250-255.

11. McGlothlin AE, Lewis RJ. Minimal clinically important difference: defining what really matters to patients. JAMA 2014; 312: 1342-1343.

12. Angst F, Aeschlimann A, Angst J. The minimal clinically important difference raised the significance of outcome effects above the statistical level, with methodological implications for future studies. J Clin Epidemiol 2017; 82: 128-136.

13. de Vet HC, Terwee CB. The minimal detectable change should not replace the minimal important difference. J Clin Epidemiol 2010; 63: 804-805

14. du Bois RM, Weycker D, Albera C, et al. Forced vital capacity in patients with idiopathic pulmonary fibrosis: test properties and minimal clinically important difference. Am J Respir Crit Care Med 2011; 184: 1382-1389.

16. King TE, Albera C, Bradford WZ, et al. Effect of interferon gamma-1b on survival in patients with idiopathic pulmonary fibrosis (INSPIRE): a multicentre, randomised, placebocontrolled trial. Lancet 2009; 374: 222-228.
17. Ware JE, Jr, Sherbourne CD. The MOS 36-item short-form health survey (SF-36). I. Conceptual framework and item selection. Med Care 1992; 30: 473-483

18. du Bois RM, Weycker D, Albera C, et al. Six-minute-walk test in idiopathic pulmonary fibrosis: test validation and minimal clinically important difference. Am J Respir Crit Care Med 2011; 183: $1231-1237$.

20. Swigris JJ, Wamboldt FS, Behr J, et al. The 6 minute walk in idiopathic pulmonary fibrosis: longitudinal changes and minimum important difference. Thorax 2010; 65: 173-177

21. Nolan CM, Delogu V, Maddocks M, et al. Validity, responsiveness and minimum clinically important difference of the incremental shuttle walk in idiopathic pulmonary fibrosis: a prospective study. Thorax 2018; 73: 680-682.

22. Swigris JJ, Brown KK, Behr J, et al. The SF-36 and SGRQ: validity and first look at minimum important differences in IPF. Respir Med 2010; 104: 296-304

23. Swigris JJ, Wilson H, Esser D, et al. Psychometric properties of the St George's Respiratory Questionnaire in patients with idiopathic pulmonary fibrosis: insights from the INPULSIS trials. BMJ Open Respir Res 2018; 5: e000278.

24. Prior TS, Hoyer N, Hilberg O, et al. Responsiveness and minimal clinically important difference of SGRQ-I and K-BILD in idiopathic pulmonary fibrosis. Respir Res 2020; 21: 91.

25. Swigris JJ, Han M, Vij R, et al. The UCSD shortness of breath questionnaire has longitudinal construct validity in idiopathic pulmonary fibrosis. Respir Med 2012; 106: 1447-1455.

26. Martinez TY, Pereira CA, dos Santos ML, et al. Evaluation of the short-form 36-item questionnaire to measure health-related quality of life in patients with idiopathic pulmonary fibrosis. Chest 2000; 117: 1627-1632.

27. Witt S, Krauss E, Barbero MAN, et al. Psychometric properties and minimal important differences of SF-36 in idiopathic pulmonary fibrosis. Respir Res 2019; 20: 47.

28. Patel AS, Siegert RJ, Brignall K, et al. The development and validation of the King's Brief Interstitial Lung Disease (K-BILD) health status questionnaire. Thorax 2012; 67: 804-810.

29. Flaherty KR, Wells AU, Cottin V, et al. Nintedanib in progressive fibrosing interstitial lung diseases. N Engl J Med 2019; 381: 1718-1727. doi: 10.1056/NEJMoa1908681

30. Sinha A, Patel AS, Siegert RJ, et al. The King's Brief Interstitial Lung Disease (KBILD) questionnaire: an updated minimal clinically important difference. BMJ Open Respir Res 2019; 6: e000363.

31. Yorke J, Jones PW, Swigris JJ. Development and validity testing of an IPF-specific version of the St George's Respiratory Questionnaire. Thorax 2010; 65: 921-926.

32. Swigris JJ, Fairclough D. Patient-reported outcomes in idiopathic pulmonary fibrosis research. Chest 2012; 142: 291-297.

33. Swigris JJ, Esser D, Wilson $\mathrm{H}$, et al. Psychometric properties of the St George's Respiratory Questionnaire in patients

\section{Suggested answers}

1. d.

2. c.

3. c.

4. a. 
Minimal clinically important difference in IPF

with idiopathic pulmonary fibrosis. Eur Respir J 2017; 49: 1601788.

34. Celli BR, Cote CG, Marin JM, et al. The body-mass index, airflow obstruction, dyspnea, and exercise capacity index in chronic obstructive pulmonary disease. NEnglJMed 2004; 350: 1005-1012.

35. Ley B, Ryerson CJ, Vittinghoff $\mathrm{E}$, et al. A multidimensional index and staging system for idiopathic pulmonary fibrosis. Ann Intern Med 2012; 156: 684-691.
36. Wells AU, Desai SR, Rubens MB, et al. Idiopathic pulmonary fibrosis: a composite physiologic index derived from disease extent observed by computed tomography. Am J Respir Crit Care Med 2003; 167: 962-969.

37. Nolan CM, Birring SS, Maddocks M, et al. King's brief interstitial lung disease questionnaire: responsiveness and minimum clinically important difference. Eur Respir J 2019; 54: 1900281. 